\title{
Decoding (patho-)physiology of the lung by advanced in vitro models for developing novel anti-infectives therapies
}

Carlos Victor Montefusco-Pereira ${ }^{2,3}$, Cristiane de Souza Carvalho-Wodarz ${ }^{1}$, Johanna Seeger ${ }^{3}$, Charlotte Kloft ${ }^{3}$, Robin Michelet ${ }^{3}$, Claus-Michael Lehr ${ }^{1,2}$

${ }^{1}$ Department of Drug Delivery, Helmholtz Institute for Pharmaceutical Research Saarland (HIPS), Saarbruecken, Germany.

${ }^{2}$ Department of Biopharmaceutics and Pharmaceutical Technology, Department of Pharmacy, Saarland University, Saarbruecken, Germany.

${ }^{3}$ Department of Clinical Pharmacy and Biochemistry, Institute of Pharmacy, Freie Universitaet Berlin, Germany.

Corresponding author: Cristiane de Souza Carvalho-Wodarz, cristiane.carvalho@helmholtzhips.de Tel: +49 (0) 681 98806-1000

Keywords: Cystic fibrosis, lung infection, respiratory research, clinical pharmacy, nonclinical PK/PD studies

Teaser: Advanced lung cell culture models provide physiologically-relevant and complex data for mathematical models to exploit host-pathogen responses during anti-infective drug testing. 


\begin{abstract}
The need for novel anti-infective therapies is dramatically increasing, not only for viral but also for bacterial infections and antimicrobial resistance. This is especially true for chronic lung infections, typically occurring during e.g., cystic fibrosis $(\mathrm{CF})$. Mimicking the structure and physiology of the diseased lung, advanced in vitro models also allow studying the pathophysiological changes relevant to microbial growth, drug resistance, and biofilm formation. Combining data from such advanced in vitro models with pharmacometric approaches may enable mechanistic explanation of the PK/PD drug exposure-response relationship and facilitate the translation to an in vivo setting.
\end{abstract}




\section{Introduction}

In 2017, the Global Burden of Diseases, Injuries, and Risk Factors Study (GBD) identified lower respiratory tract infections as the $4^{\text {th }}$ leading causes for early death worldwide, accounting for more than 1 million deaths annually. This category includes pneumonia, bronchitis, and viral infections, and this death count is disproportionally higher for children and adults over 70 years[1]. Lung infection also has crucial implications on lung physiology that challenges the way we develop and test new anti-infectives.

Bacterial growth results in the release of virulence factors that change the lung physiology. Acute infection can lead to cell and tissue destruction and develop into a chronic stage characterized by biofilms formation[2]. The severity of infections is increasingly worsened by antimicrobial resistance (AMR), which is believed to become a major cause of death by 2050 [3]. Also, the expectation that antibiotics shall cure disease and not only alleviate symptoms poses a particular challenge to the pharmaceutical industry.

The primary innate defense mechanism in the lungs against inhaled particles and pathogens is the mucociliary clearance. Additionally, trachea-bronchial mucus and the alveolar lining fluid with the lung surfactant and alveolar macrophages are natural clearance factors that continuously remove inhaled particles. A recent study showed that formylated bacterial peptides stimulate the release of acetylcholine from tracheal cells, leading to increased ciliary beating and thus mucociliary clearance[4]. It still needs to be investigated whether such mechanisms also apply to non-bacterial particles. Indeed, the pulmonary lining fluids are a structural barrier protecting the inner tissue while containing antimicrobial peptides to exert action locally. The lung epithelial and endothelial layers provide a physical barrier against particles and microorganisms to access the bloodstream. In such a scenario, the resident immune cells scan for every particle and, when needed, produce danger signals to recruit the immune system to act. This interplay between host and microbes and its modulation by antimicrobial therapy is observed in several respiratory diseases, for instance, asthma, chronic obstructive pulmonary disease (COPD), and cystic fibrosis (CF). In this review, we will mainly focus on infectious diseases with a particular focus on $\mathrm{CF}$ - a genetic disorder that alters the flow of chloride into the cells. In the lung, CF causes alterations that facilitate the establishment of chronic infections[5].

If a new anti-infective has potential against respiratory infections, the drug testing starts with pre-clinical studies involving cell culture and animal models. In the case of CF, advanced in vitro cell culture systems have been used to emulate $\mathrm{CF}$ disease and host-microbe interactions[6]. Such models allow evaluating the efficacy, but also to identify the hurdles a 
drug might face during its deposition or interaction with biological barriers and matrices. Furthermore, advanced in vitro models may help to define the next step in drug development, including providing insights for drug dosing regimen and mechanism of action, and thus to optimize their delivery to the target. However, the establishment of such in vitro systems and their validation to mimic human physiology remains challenging[7].

A multidisciplinary approach is needed to tackle the analysis of complex data arising from such in vitro systems, combined with the compound's kinetics and its direct influence on the human physiological response, and the host-microbe-drug interplay. The field of pharmacometrics, which relies on data from (pre-)clinical trials, generates mathematical models to explore multiple scenarios for the drug action and interaction on the body as well as the elucidation of pharmacokinetic-pharmacodynamic relationships enabling a translation of data integration[8].

Nonetheless, some steps still need to be made to use pharmacometrics to bridge the gaps between current in vitro models and in vivo situation. A first example is that the investigation of drug efficacy is currently performed on bacteria grown on abiotic surfaces (i.e., plastic supports). The integration of the epithelial cell layer and immune cells into this surface would increase the complexity of the in vitro system to mimic a living substrate for biofilm development and further drug testing[9]. A second example would be the study of immune cell recruitment and host response, which has also been investigated using pharmacodynamics modeling studies.

Another example involves studies of bactericidal activity, which quantify the concentration-effect relationship of a new drug. However, the majority only report static concentrations as minimal inhibitory concentration (MIC). At the same time, a time-kill curve experiment could be used to build a mathematical model to simulate all possible scenarios of the host-microbe-drug interplay[10]. In a dynamic time-kill curve approach, the drug concentration can vary over time using a pumping system, and this study is a more clinically relevant approach that both fields can benefit from the building of pharmacokinetic/pharmacodynamics (PK/PD) modeling [11]. In this review, we highlight the need for bridging in vitro pharmacodynamic experiments to develop useful in vitro models of long-term pulmonary infections. Such an approach has the potential to provide new scientific data that could help to predict drug efficacy and drug kinetics from in vitro to in vivo, in a coherent and translational framework. 


\section{Lung physiology and drug delivery}

The conducting airways of the central lung and the respiratory epithelium of the peripheral lung are the main constitutes of this organ. Once inhaled, the air is humified and filtered in the conducting airways - this area is comprised of the nasal cavity, mouth, trachea, bronchi, and bronchioles. Respiratory bronchioles, alveolar ducts, and sacs compose the respiratory region of the lung [12].

While the alveolar epithelium of the deep lung is much larger $\left(100-140 \mathrm{~m}^{2}\right)$, the airways have a relatively small surface area (1-2 $\left.\mathrm{m}^{2}\right)$; nevertheless, the airways represent the primary target as well as a biological barrier for the majority of inhaled particles (including drugs) and microbes. The airways provide multiple defense strategies, such as mucociliary clearance, the maintenance of electrolyte levels, epithelial lining fluid, and mucus that releases antiinflammatory, anti-infective, and antioxidant molecules. Next to the chemical protection, there is a second line of defense: an epithelial barrier developed by relatively tight cell-to-cell connections. With the introduction of allergens or microbes (inhaled or from the bloodstream), the barrier can be compromised as a result of direct cytotoxic effects or inflammatory reactions[13].

The pulmonary surfactant, produced by AT2-cells of the alveolar epithelium, provides additional barrier functions, such as reducing surface tension and antimicrobial activity. As a major part of the surfactant, DPPC, and the lung surfactant proteins (SP) are most efficient in lowering the surface tension of the pulmonary lining fluid. A surface tension lower than 1 $\mathrm{mN} / \mathrm{m}$ prevents the lung from collapsing and facilitates breathing. Besides, the surfactant proteins SP-A and SP-D are a particular group of lectins, so-called collectins, that target viral and bacterial glycans via their C-terminal to promote macrophage and monocyte-mediated phagocytosis [14]. Cellular models provide the building blocks to study lung physiology in health and disease. While animal in vivo models are key tools to comprehend the alterations in the respiratory tracts, translation to the human system is limited by species differences that may be better resolved by the human in vitro models. For example, on the lung structure, the use of genetic gain-and-loss-of-function studies report the importance of signaling pathways in the development of the lung, including how branching of the lung tree bifurcations start. Nevertheless, rat and mice models are used to understand lung injury that is comparable to humans as fibrosis and emphysema[15].

In contrast, the translation of these results to the clinic remains a challenge. Parameters in the development of the mouse lung are faster than in humans; moreover, the mouse only develops alveoli after birth. Furthermore, on the apparent difference of size and branching 
steps, the rodent lung also presents alternative sites of progenitor and stem cells. These distinctions will lead to $80 \%$ of failure on drug efficacy in human trials from molecules that were previously tested on the rodent lung [16].

Therefore, there is a need for suitable human cell models that could better predict drug efficacy before animal and human clinical trials. Moreover, in vitro models can provide largescale screening platforms, assessment of specific cell-to-cell molecular differences, and the opportunity to understand lung disorders while providing insights into molecular mechanisms that cause variability in drug efficacy between individuals.

\section{In vitro models of the lung: what to consider regarding aerosol deposition?}

The pulmonary route allows both for local-regional delivery as well as systemic drug delivery. It is essentially non-invasive - an advantage to parenteral delivery (i.e., by injection). In the context of pulmonary delivery, human cells with their intrinsic biological matrices, genetic specificity, metabolic enzymes, efflux pumps, mechanisms of clearance, and biological barriers, may allow to step-wise address the lung's complexity[16]. Parameters like lung morphology, aerosol characteristics, target disease, besides pharmacokinetics and pharmacodynamics, should also be considered in the aerosol deposition.

The development of inhalation formulations assumes that with the total delivered dose, also the regional deposition is possible. The inflammation observed in the pathophysiological characteristics of COPD and asthma is a drawback to be overcome by formulations - the latter should adapt their deposition dynamics to reach the peripherical airways. Also, targeting the alveolar region is used for more efficient drug absorption in the case of systemic delivery. In the clinics, the study of albuterol aerosols against asthma, through $\mathrm{FEV}_{1}$ in spirometry evaluation, showed that $6 \mu \mathrm{m}$ particles were associated with improved bronchodilation, when compared to 3 and $1.5 \mu \mathrm{m}$ particles. Such effect was due to the relative selectivity of the proximal airways in the bronchial tree (the target of bronchodilators), while the smaller particles went deep into the lung[17]. In general, particles with a mass median aerodynamic diameter (MMAD) of 5-10 $\mu \mathrm{m}$ will use gravitational sedimentation and be allocated at the middle or upper airways. In contrast, MMAD of 2-5 $\mu \mathrm{m}$ reaches the middle or deep airways. Using Brownian motion, particles with MMAD under $2 \mu \mathrm{m}$ will target the small airways and alveolar region[18].

Classic in vitro models (i.e., submerged cultures) of bronchial or alveolar epithelial cells are widely used to assess permeability as well as direct local effects of inhaled pharmaceuticals. While this approach is fast and has low costs for routine experiments, the cell exposure to the 
air, so-called air-liquid interface (ALI), represents a more accurate depiction of the human respiratory in vitro. The cultivation of cells on porous membrane inserts allows working under ALI conditions, with feeding and humidification being provided through the basolateral side[19]. Some exposure systems for ALI in vitro models have been described in the literature or are already commercially available, for instance, TSE Systems (Bad Homburg, Germany), Vitrocell (Waldkirch, Germany), CULTEX (Hannover, Germany) or Pharmaceutical Aerosol Deposition Device On Cell Cultures (PADDOCCs)[19,20].

Compared to other routes of administration, the development of aerosol medicines, however, imposes additional challenges. The pathophysiological changes of the lung function in diseases like COPD or asthma may affect both pulmonary deposition as well as clearance of inhaled aerosol particles. Besides, drug solubility, release, aggregation, particle size, and chemical alterations are important biopharmaceutical parameters that must be accurately controlled and characterized[19].

The mucus layer covering the upper airways (as a hydrogel of more than $50 \mu \mathrm{m}$ thickness) is very effective in trapping and expulse particles via mucociliary clearance. The same is true for the pulmonary surfactant (an $80 \mathrm{~nm}$ layer) produced in the alveolar region that also covers mucus in the airways. If a given drug or nanoformulation is not soluble, the particles can be quickly engulfed by resident macrophages. This impacts the local drug delivery, as well as systemic bioavailability, since these biological barriers and epithelial permeability may limit transport to the bloodstream [21].

\section{A brief overview of pulmonary in vitro models}

Cell- and tissue-based in vitro models are of increasing relevance to pulmonary drug delivery to mirror the peculiarities of the respiratory system[16]. Stable cell lines, primary cells from human donors, or even human stem cells can be used to reconstruct lung tissue. In fact, in vitro cell models are a relatively new concept to mimic different aspects of the diseased lung and to develop new platforms for drug discovery and drug testing. As primary cell culture is demanding and expensive, the use of immortalized cell lines is often preferred. Some cell lines, derived from well-defined parts of the lung, eventually even in the disease state, combine the stability of infinite proliferation with the advantages as the preservation of cell morphology and functionality[22].

The variety of more than ten cell types, ciliary structures, surfactant fluids, and mucus poses a challenge for emulating the in vivo airway system in vitro[23]. Therefore, several 
groups have established co-cultures models, usually constituted of two or more cell types $[24,25]$. This combination goes beyond the traditional cultivation of cells as monocultures, allowing access to increased inflammation, excessive immune cell recruiting, tissue damage due to matrix-breaking molecules, and failure of bacterial control[26].

Human primary bronchial epithelia can be obtained through biopsies and explants. However, cell yield, bacteria colonization from the host, and lung injuries turn the procurement of primary cells into a challenge[27]. Other options, such as airway brushings or bronchoalveolar lavage and nasal swabs are being used, although cell counts are scarce in CF patients[28]. New technologies access the development of patient-specific cells, including basal stem cells, trans-differentiation (percent of alveolar surface area covered by one cell type) from type-II alveolar into type-I cells, and inducible pluripotent and embryonic stem cells (Fig. 1A)[29,30].

An overview of the in vitro models and possible applications towards drug efficacy testing, aerosolization, and PK/PD relationships is provided below and in Table 1.

Static systems. Here the cells are grown either on standard culture plates or permeable membrane inserts. For controlling the deposition of aerosols, such models ask for ALI conditions, as shown, for instance, by Ong et al. [35]. Here, ciprofloxacin was delivered as an aerosol, and its cellular transport through epithelial barrier assessed. In the future, such an approach could consider pharmacokinetic alterations due to pharmaceutical formulations. Kletting et al.[22] also delivered silver nanoparticles and investigated their pulmonary and immune toxicity. Mostly parameters, such as macrophage migration or nanoparticle uptake, are already part of the system's general characterization. Besides, it may deserve attention to how such parameters could also be used in PK/PD. For instance, with the nanoparticle uptake by macrophages, the measured uptake rate can be considered when evaluating drug transfer, absorption, and possible clearance.

Microfluidic systems. These are devices specifically designed to emulate human (patho)biology in vitro, sometimes also referred to as organs-on-chip. A systematic report on the potential of microphysiological systems for drug development and animal welfare has been recently published [36]. Sophisticated lung-on-a-chip systems integrate advanced cell culture into microfluidic channels and chambers, aiming to engineer structures capable of representing the air-blood barrier. This concept has been integrated into a differential application of flow and even stretching mechanisms similar to lung movements during breathing. More recently, such mechanical factors have been implemented to study pulmonary edema and the role of 
interleukin-2, or the influence of interleukin-13 on ciliary beating, recapitulating processes from chronic obstructive pulmonary disease (COPD) patients[16]. Other groups built chips based on morphometric measurements of the adult human lung, adding human bronchial cells in ALI conditions for 21 days, where cell differentiation is observed towards the detection of mucus and ciliated cells. Here, the addition of flow and shear could gather influence on drug biokinetics and allow assessing immune cell recruitment [37].

Organoids. Lung development has been explored with so-called bronchospheres, which are organoid-like cultures that integrate basal stem cells embedded into an extracellular matrix. They form spherical colonies with secretory ducts and ciliated cells. Furthermore, the coculture of bronchial cells with endothelial cells and fibroblasts provided cues to form lung capillary structures. Moreover, lung cells from CF patients were grown as organoids and infected by the respiratory syncytial virus, as a representation of the infected CF lung[38]. Human pluripotent stem cells cultivated in alginate beads and collagen in a bioreactor were able to form organoids similar to alveolar sacs.

Nonetheless, the organoids functionality as "mini-lung" is still to be improved. Bronchial epithelial cells, lung fibroblasts, or endothelial cells can be cultivated as airway organoids to stimulate plasticity with proximal cell interaction and microenvironment in coculture [16]. Expanding this concept towards a multi-organ chip, Bovard et al. combined lung and liver cells in the same system[34]. The potential of aerosolization was explored using pollutants and testing lung toxicity and liver metabolism. This combination can provide future insight into drug biokinetics and $\mathrm{PK} / \mathrm{PD}$, such as clearance and biotransformation, while mimicking pollutants or drug delivery via the lungs. Also, compartmental clearance can be considered for different organs as a pre-evaluation for a future physiology-based pharmacokinetic (PBPK) model.

Ex vivo models. These models are somewhat challenging to handle (e.g., an isolated perfused lung), but have the advantage to represent the whole organ inside an artificial environment with minimum alterations. Here drugs, particles, and pathogens are parameters to be considered to increase physiology and model complexity. Alternatively, slices of the lung can be cultivated. These approaches preserve not only the in vivo architecture, but also the cell types enclosed. Multiple cellular functions can be demonstrated, including adequate mucociliary clearance in the embryonic chicken trachea, independent of particle size or shape. Models such as the porcine lung show a spatial structure very close to the human lung[15]. Here, the concept of 
bound versus unbound drug fraction is used to adapt experiments on the binding to plasmatic proteins, such as albumin. Besides, clinical parameters, like lung volume, ventilation rate, and respiratory patterns, could be simulated with the control of flow in the organoid model.

\section{Critical issues of in vitro models for pulmonary drug development}

Sakagami[39] reviewed different lung models used for testing inhaled drugs and addressed that the choice of model is intrinsic to the scientific question. In vitro models can indeed be a sophisticated tool to minimize the complex interactions of living organisms, while allowing studying intercellular interactions within the lung, as well as the effect of inhaled particles or pathogens.

As mentioned before, when considering pulmonary deposition, in vitro models should be exposed to the air to allow for aerosolization. In fact, the use of cultures at ALI is a standard method in respiratory research. Although some difference between in vitro models at ALI and direct human airways derived from bronchoscopy or bronchial brushing could be founded e.g., the expression of genes related to cilia formation - other features did not show a significant change: genes related to ion transport, antimicrobial peptides (excluding lysozyme), surfactants, and cell adhesion molecules[40].

In the intact human lung, the process of drug absorption is important to be evaluated. In vitro, cell layers that form tight junctions can be used to understand drug permeability in the lung. The Calu-3 bronchial immortalized cell line has been used to understand the roles of passive drug diffusion or interaction with OCT or P-gp transporters. For that, the apparent permeability coefficients ( $\mathrm{P}_{\text {app }}$ values) of in vivo models can be compared with $\mathrm{P}_{\text {app }}$ values from in vitro epithelial barrier. Coculture models offer a more sophisticated approach to assess particle uptake and cell-cell interaction (e.g., lung epithelial and immune cells) [27] as well as the drug efficacy on an infected bronchial epithelium [44]. The latter system allows for simultaneous measurement of inflammation, epithelial barrier integrity, macrophages transmigration, and $P$. aeruginosa survival. Those parameters are essential for monitoring drug efficacy and host response and shall be useful in the test of new anti-infectives.

Advanced in vitro complexity allows for better exploration of physiological and cellular responses. Using well-plate inserts, advanced coculture models have been described to mimic the interaction between different lung cells. The pulmonary epithelium, bronchial or alveolar, is always present as the main barrier, which can be complemented by other cellular components, including immune cells. For instance, the combination of bronchial with endothelial cells[42], alveolar cells with fibroblasts[43], airways with neutrophils[44], and 
bronchial cells with alveolar macrophages[41,45] and also endothelial cells[46] has already been reported. Some commercialized models can reach full lung differentiation, as e.g., in MucilAir ${ }^{\mathrm{TM}}$ (Epithelix, Suisse) with immune cells, enabling the understanding of pathology, inflammation, and host response, mainly due to the presence of mucociliary clearance. In cocultures, an alveolar-capillary barrier can be integrated to study the toxicity of materials such as silica nanoparticles[47]. Chowdhury et al.[48] observed an increased mRNA level of the tight junction protein, occludin, when bronchial epithelial and endothelial cells were cultivated together. Even without immune cells, cocultures can shed light on several aspects of inflammation triggered by particles. For instance, a coculture of alveolar epithelia with pulmonary endothelial showed increased barrier function but also increased IL-6 and IL-8 expression during the exposure to the environmental pollutant cadmium $\left(\mathrm{Cd}^{2+}\right)[49]$.

\section{Modeling the lungs in state of diseases}

Lung development and homeostasis are prominent and challenge areas to be emulated in vitro. The high global burden of infectious lung diseases and drug failure ask for innovative therapeutic modalities. In this context, there is an urgent need for the development and validation of in vitro models that allow multiple readouts options and better emulate the physiological changes observed in vivo. From primary to immortalized cells, in vitro models enabled long-term cell culture with the advantages of cell differentiation and possibilities to develop scenarios of disease progression, including acute to chronic infection[50].

Lower respiratory lung infections like chronic obstructive pulmonary disease (COPD), Tuberculosis, and Pneumonia share the common drawback of antibiotic failure and bacteria resistance. Moreover, the currently challenging with Coronavirus (COVI-9) infections stress the need for appropriated lung disease in vitro models.

COPD. Chronic airway inflammation is the main trigger of COPD. In vitro cell models have also been applied to uncover new COPD targets, for instance, the use of COPD bronchial epithelial and lung fibroblasts cells to show TGF- $\beta$ as a target against lung fibrosis mediated by squamous bronchial cell metaplasia[51]. Also, COPD patients are known to be affected by infections with high microbial diversity (even anaerobes pathogens)[52]. This model may allow the assessment of the synergic effect of anti-infectives with anti-inflammatory or immunomodulatory drugs. Another COPD in vitro model mimicked Goblet cell hyperplasia, a characteristic of COPD triggered by particulate matter from cigarette smoke. For that, primary human bronchial epithelial cells (HBEC) were exposed to cigarette smoke for 28 days leading to an increased expression of MUC5AC positive cells on the overproduction of mucus [53]. 
Pneumonia. This is a disease affecting the alveolar region, with inflammation and infection being the main hallmarks. Several microbes could be responsible for pneumonia, including Streptococcus pneumoniae, Haemophilus influenzae, and Acinetobacter baumanii. For that, in vitro models have been developed to understand the host-microbe interplay. For instance, a study with $S$. pneumoniae severely altered the cytoskeleton of ciliary cells in the lung[54]. Moreover, a 3D coculture model combined bronchial epithelial cells, fibroblasts, and neutrophils helped to assess the effect of $S$. aureus alpha-toxins or PVL toxin itself on the disruption of the lung barrier[55].

Tuberculosis. This is another challenge disease to mimic in vitro. This infection is caused by Mycobacterium tuberculosis, and it requires compliance of two years to a therapy regimen involving drugs leading to adverse effects. In vitro models can now be used to mimic the early stages of granuloma formation with human lung cells, fibroblasts, leukocytes, and the microbe itself[56]. In recent model development, lung cells could be encapsulated into alginate microspheres to mimic granuloma formation[57]. This allowed studying the influence of collagen on the granuloma matrix and the immune response of encapsulated cells when stimulated by Mycobacterium tuberculosis-responsive T cells.

COVID-19. This is a recently pandemic virus infection characterized by acute respiratory disease, caused by the 'severe acute respiratory syndrome coronavirus 2' (SARSCov-2). The urgency for new therapy against this virus asked for the development of several in vitro and in vivo models[58]. In vitro models are particularly important here because they allow for a better understanding of the virus biology and pre-clinical evaluation of new therapies. Although cell lines from different organs have been used in COVID-19 research, the epithelial cells from airways are the ones expressing receptors used by the virus to infect cells (angiotensin-converting enzyme 2 and transmembrane serine proteinase 2). For instance, Calu3 lung cells showed an increased infection rate of SARS-CoV-2 virions. Lung organoids derived from stem cells allowed the observation that type-II alveolar cells are more prone to be infected to the SARS-CoV-2. Moreover, human bronchial organoids showed the presence of viral genome integration, pyknotic cells, and stimulation of Type-1 IFN response; besides, this model was used for drug testing[58].

\section{In vitro models of the infected lung in the context of $\mathrm{CF}$}

$\mathrm{CF}$ is characterized as a genetic autosomal recessive disorder caused by mutations in the cystic fibrosis transmembrane conductance regulator (CFTR); the latter is an ABC ion channel transporter of chloride, regulated via cyclic adenosine monophosphate (cAMP). CF 
can affect organs such as the pancreas, liver, intestine, and lungs - the latter through significant airway obstruction[59]. Recent progress has allowed the expansion of patient-specific lung cells with the development of inducible pluripotent stem cells (iPSC)[60], allowing for the elucidation of the full genetic background related to the disease. Here personalized medicine and research on gene targets and repair, including mutations correlated to $\mathrm{CF}$, could be applied. Nonetheless, to create iPSC models, one needs a batch of samples obtained from different patients, which could result in increased variability due to differences regarding age, sex, disease severity, and other pathophysiological variables.

With CF providing a complex scenario, there is now the opportunity to create new platforms for drug testing, starting by emulating the disease, infection, and consequences in lung cell models (Figure 1). Direct cultivation of cells from the trachea, bronchi, and alveoli is possible with advances in cell culture and access to primary cells (Figure 1A). However, the re-building of epithelial layers with primary cells needs to mimic the lung environment, including the air-to-blood interface. Advancing these models, the inclusion of immune cells (Fig. 1B and C) has now provided differences between non-CF versus CF cells (NuLi versus CuLi-1 with $\triangle F 508$ CF mutation) with an increase in inflammation in the presence of CFTR defects, including increased neutrophil migration[61].

Cell models of the bronchial area can now be cultivated at the ALI, in double to triple cell-culture, with the possibility to include extracellular matrices and shear stress (Fig. 1D and 1E). This provided insights into the characterization of CF immortalized cells, including CFBE410 ${ }^{-}$cells, which intrinsically express the deletion of CFTR via the $\Delta$ F508 mutation, mature receptors such as TLR1-5 and TLR1-9 as well as healthy bronchial cells such as 16HBE14o[62]. Coculture with immune cells has been used not only for the analysis of drug delivery methods but also on CF infection like the inclusion of $S$. aureus $[9,22,63]$. This opens the possibility of mimicking and investigating CF co-infections as opportunistic infections by pathogens, such as $P$. aeruginosa with $S$. aureus, B. cenocepacia, H. influenzae, or the respiratory syncytial virus (Fig. 1F).

Besides the impact on the host, the outcome in CF disease will also be influenced by the bacterial growth, more specifically by the transition from planktonic to biofilm form. Marquis-Moreau et al.[64] have demonstrated the use of cocultures to observe early biofilm formation on top of CFBE410 ${ }^{-}$cells after six h of $P$. aeruginosa infection. This model increased understanding of biofilm control, drug efficacy, and antibiotic resistance. Transmigration of immune cells in vitro, focusing on the Staphylococcus aureus [9] and Pseudomonas 
aeruginosa[41] infections have also been developed. The latter in vitro system was established at ALI to enable controlled deposition of aerosolized drugs and nanocarriers.

While early $P$. aeruginosa colonization of the lung can now be mimicked in vitro, the establishment of a mature biofilm on epithelial cells is still a challenge. The infection itself is highly toxic to epithelial cells, reducing their viability within 6-8 hours of infection[64]. This limits further studies on bacteria-host cell responses in chronic infection or repeated dosage regimens. Thus, different strategies are being used, such as the external growth of biofilm and transfer of its content, or the combination of biofilm growth on a substrate composed of airway cells or a polymeric scaffold (Fig. 1E)[65].

The combination of cells and pathogens allows for increasing the complexity of the system, more closely resembling the in vivo condition. Some cells command the immune reaction, while others create a biological barrier, and both elements are relevant to pathogen adaptation and growth. Although in vitro models exist and are advancing in required complexity, we shall take advantage of concepts from the field of toxicology, for instance, the Quantitative in vitro to in vivo Extrapolation (QIVIVE). Toxicologists evaluate risk assessment by focusing on literature, physicochemical reactivity, drug biokinetic, effect battery test, concentration responses test, and considering population variability and sub-populations [66]. This concept should be in the future applicable to anti-infectives and pulmonary delivery. This is because crucial factors can contribute to efficacy or interaction assessment, as:

- macrophage cells may interfere with particles and drug concentration;

- the epithelial layer may act as a barrier for drug products, and this will determine lung permeability;

- the chemically and electrostatically characteristic of bacterial biofilm may repulse drug particles, hampering thus their action on bacteria;

- the contact of bacteria with the lung epithelia may induce the release of virulence factors to promote bacterial adherence and the formation of bacterial communities;

- ventilatory frequency in different individuals of different disease stages may require more effort or drug during inhalation delivery.

These parameters, at the moment, are qualitative, which ask, therefore, for the production of quantitative data. Adequate in vitro models are necessary for the evaluation of pharmacokinetics parameters. There is great potential, for instance, in aerosolization studies, 
for the generation of crucial data that may contribute to the development of pharmacometrics models. Addressing those points, we compiled the association of PK/PD, aerosolization, and drug efficacy on in vitro bacteria-infected systems (Table 2).

\section{PK/PD aspects of bacterial infections and anti-infective therapies}

In vitro models provide complex data that can be exploited regarding the effect of a drug in the body (pharmacodynamics-PD) and the influence of the body on the drug (pharmacokinetics-PK). PK/PD characterization of the relationship between antibiotic, host, and pathogen can elucidate the variability of responses that could be expected in a human population.

The introduced in vitro lung models are capable of mimicking pathological alterations of the lung in $\mathrm{CF}$, infection, and immune response. However, they typically do not account for the PK/PD of an antibiotic. This is determined by the physicochemical properties of the compound and (patho)physiological characteristics of the host, resulting in individual antibiotic concentration-time profiles at the target site. The PD effect of an antibiotic can be quantified by assessing serial bacterial concentrations over time in PK/PD in vitro models[75]. Thereby, linking drug exposure to the resulting bacterial growth and killing dynamics provides insights into the complex interplay between antibiotic, host, and pathogen.

In static in vitro models, constant antibiotic concentrations are applied, while dynamic models mimic in vivo concentration-time profiles, resulting from the pharmacokinetic processes in the host[11]. Assessing the bacterial growth and kill trajectory enables a comprehensive characterization of the PK/PD relationship by applying metrics such as the relative bacterial reduction, representing the drug effect more comprehensively than the commonly used MIC[76]. Additionally, PK/PD indices, such as the maximum concentration $\left(\mathrm{C}_{\max }\right) / \mathrm{MIC}$ ratio or the area under the concentrate-time curve (AUC)/MIC ratio can be derived when the drug concentration-time profile is assessed in a dynamic in vitro model. Based on that, the required target values for the desired effect, such as bacterial eradication or a specific reduction of bacterial load, can be determined[77].

Based on in vitro time-kill curve experiments, in silico, PK/PD models can be developed. By linking applied drug exposure to the observed antibacterial effect, these models aim to predict the bacterial growth/kill trajectories based on MIC values, genomic resistance mechanisms, or measures for phenotypic adaptation, such as for persistence cell formation, indicated by bacterial filamentation[78]. 
Bacterial subpopulations, such as susceptible, resistant, and antibiotic-tolerant bacteria, can be quantified by estimating bacterial growth and death rate constants, as well as transfer rate constants between replicating and non-replicating subpopulations, providing mechanistic explanations for observed bacterial killing and regrowth under antibiotic exposure[79]. Experimental strategies to assess adaptation and resistance mechanisms are warranted to inform these mechanistic PK/PD models and enable translation into an in vivo setting. An interesting approach was presented by Thorsted et al., who determined endotoxin release of Escherichia coli under static and dynamic cefuroxime exposure and linked it to bacterial growth and antibiotic-induced killing of susceptible and filamentous bacteria[80].

Leveraging mathematical modeling to exploit complex data generated in PK/PD in vitro models can elucidate bacterial adaptation mechanisms and the emergence of resistance under antibiotic exposure, but typically lacks the immune response of the host. The pharmacometric approach offers an opportunity to amalgamate insights from cell culture, PK/PD in vitro models, and clinical studies to optimize antibiotic therapy for individual patients ultimately.

\section{Expanding pharmacometric approaches towards pulmonary anti-infectives}

Comprehensive assessment of data on absorption, distribution, metabolism, and elimination is a prerequisite for the mathematical characterization of the PK/PD relationship of a drug and a pathogen, which is enabled by applying the techniques of quantitative systems pharmacology (QSP) and pharmacometrics[81].

$\mathrm{PK} / \mathrm{PD}$ investigations aim to relate clinical cure to drug exposure via metrics measuring in vivo and in vitro microbiological eradication (from microbe inhibition - MIC, bactericidal effect, MBC, and biofilm inhibition or eradication - MBIC/MBEC[82] and dynamic time-kill curves). For the testing of anti-infectives, the antibacterial activity can be classified as either time or exposure dependent. However, clinical trials require a decision on a specific dosing regimen, for instance, during dose-fractionation studies. This evaluation is typically done in animal models withholding markers of efficacy as decreased bacterial concentrations (log $\mathrm{CFU} / \mathrm{mL}$ ) in animal tissue might correlate to the clinical efficacy of a drug. An earlier step on the definition of dose range is done by in vitro studies. The MIC is determined in vitro as the lowest concentration that inhibits visible bacterial growth using 2-fold dilution steps of an antibiotic. Different bacterial species with clinical relevance are investigated in vitro, resulting in specific MIC distributions for a species. Both laboratory reference strains, provided by the American Type Culture Collection (ATTC) and clinical isolates, are investigated, elucidating mechanisms of drug resistance. For that, in vitro testing can start with one microbial species to 
evolve into genetically-different strains to infection on live substrates or scaffolds or advanced cell to organoid models[83].

The most common PK/PD indices relate the percentage of the dosing interval in which the unbound drug concentration (considering protein binding) exceeds the MIC $\left(f \mathrm{~T}_{>\mathrm{MIC}}\right)$, the maximum concentration at the target site divided by the $\mathrm{MIC}\left(f \mathrm{C}_{\max } / \mathrm{MIC}\right)$ and the $24 \mathrm{~h}$ exposure to the drug over the MIC (area under the unbound drug concentration-time curve fAUC/MIC)[84]. For inhaled drugs, some indices can be obtained from inhalation studies, for instance, in the animal model to perform histopathology on the observation of interstitial necrosis and the presence of pro-inflammatory cells[85]. Nevertheless, from human trials, the clinical endpoint of respiratory strength is important, as FEV1, FEV1\% predicted, forced vital capacity, forced expiratory flow between $25 \%$ and $75 \%$ of forced vital capacity (FVC), and vital capacity. This has been exemplified on an established PK/PD model towards inhaled liposomal amikacin to CF patients[86].

This approach is being acknowledged as part of the regulatory process for new drug approvals, with the European Medicines Agency providing a guideline on how to apply the PK/PD models. This guideline requests leveraging time-kill curve experiments to inform mathematical models, which can be used to define target values for the PK/PD indices, by simulations. Based on the probability of target attainment analysis (PTA), the selection of dosing regimens is supported. One strength of this approach is the individualization of dosing regimens to patient subpopulations, such as patients with impaired renal function, pediatrics, elderly, obese, or critically ill patients[87].

The evolving field of pharmacometrics has contributed to a more comprehensive understanding of complex systems. The concept of mechanism-based PK/PD models addresses how biological (and pathological) processes may directly influence the pharmacokinetics and resulting pharmacodynamic effects. The human body and the microbiome are in homeostasis in which the involved elements undergo an inevitable turnover. This holds for the cells and organs of the human host, and secreted hormones or neurotransmitters, but also for bacteria and their secretions, in particular virulence factors. So, when PK/PD indices are increasingly variable, one option is to integrate microbial growth kinetics. In the future, the integration of data from other processes as disease progression or immune reactions should be foreseen. The mechanism-based PK/PD modeling approach allows for the characterization of bacterial growth behavior under antibiotic exposure based on in vitro or in vivo data by Jusko[88] and Rathi et al.[89]. 


\section{Bridging drug delivery and pharmacometrics}

Drug delivery via the lung is complex, involving several competing processes that can be described by pharmacometric approaches[90,91]. In the case of bacterial lung infections, the underlying processes are drug deposition, interaction with lung fluids, lung clearance mechanisms such as mucociliary clearance and macrophage uptake, lung retention of a drug, and its metabolism and transport towards other tissues or biofilm[90]. By stimulating cell growth and differentiation, features such as lung mucus or surfactant can advance in vitro systems to assess the behavior of inhaled particle or aerosol in the different parts of the lung. This was exemplified with a cellular pharmacokinetic model that explains drug accumulation due to passive transport in areas as the apical, basolateral, or intracellular regions[92]. Not only drug delivery into the lung should be accounted for, but also the different pathways taken by a drug to reach its target site, which is typically investigated using pharmacometric approaches.

In such a multidisciplinary approach, doubts concerning which dosing regimen to start therapy can consider the clinical breakpoints determined by the EUCAST[93]. Susceptibility breakpoints can then directly help to treat infections. This determination is achieved when relating $\mathrm{PK} / \mathrm{PD}$ indices, however, attentive to the target site and its variations of drug penetration, for instance, in biofilm, from its formation to eradication[94]. There are few examples for the use of pharmacometrics modeling based on in vitro and pre-clinical data in pulmonary therapeutic settings focussing on biofilm penetration, which are summarised in Table 3.

These examples of multidisciplinary research represent the first approaches to combine pulmonary drug delivery and pharmacokinetic models in the context of bacterial biofilms. However, an overarching model taking all processes into account, as depicted in figure 2 , is still lacking. The examples in Table 2 suggest that a multidisciplinary collaboration between pharmacometricians and pre-clinical scientists in the field of pulmonary biofilms could lead to the extraction of more knowledge from both in vitro and in vivo studies. However, the number of experimental datasets analyzed by these advanced techniques remains low. Optimal design theory based on these models could be applied to streamline the flow of information and efficiently design these experiments.

Eventually, pharmacometric models can link antibiotic dosing to resulting concentration-time profiles at the target site and the therapeutic effect of the drug. Moreover, different mechanisms of acute or chronic infection could elucidate factors that can reduce antiinfective resistance and provide insights on appropriate dosing regimens for diverse populations. 
540 Concluding remarks and future perspectives

541 Both established as well as emerging therapeutic approaches to pulmonary infections 542 require novel tools for their fast and efficient development. In this review, we presented the 543 lung as a challenging target for drug administration and innovative in vitro and in silico 544 techniques, providing mechanistic insights in pathologically relevant processes. In the 545 development of anti-infectives, finding new treatment options and mechanisms of action is 546 crucial. Nevertheless, increased failure has called for technological advanced approaches[100].

547 The different microenvironments of the respiratory tract (nasal, trachea, bronchial, alveolar regions), containing different cell types and functions, enzymes, immune cells, and lining fluids are especially challenging regarding drug physico-chemistry[90]. Moreover, intracellular infections in macrophages, using lung structures to build communities, are challenging drug targets. Pathogens causing these infections take advantage of mucus and dead neutrophils to create biofilm or use the lung as a gateway into the bloodstream[101].

We discussed how human cell culture systems might be adopted to reproducibly model the relevant (patho-) physiological characteristics for drug delivery, mimicking the lung as a living substrate, barrier, or target. In the case of CF models, for instance, the cell culture of the bronchial epithelium is combined with an endothelial layer and immune cells to understand structural conditions for drug administration and action. However, these integrated experimental models may also be challenged with microbial growth of $P$. aeruginosa, mimicking acute infection, as well as virulence factors disarming therapy.

Complementary, pharmacometrics exploits in vitro data and data from (pre-)clinical trials to develop mathematical models that characterize and quantify the kinetics of an antibiotic drug and its effects. They can be applied for simulations of different dosing regimens and administration routes for various compounds in mono- or combination therapy or to quantify and explain sources of variability in diverse patient populations. Communication among scientific communities provides the opportunity to exploit complex data and develop novel approaches, amalgamating different fields of expertise. The combination of pharmacometrics and pre-clinical pharmaceutical sciences offers unique opportunities to understand and explain complexity, provides insights into the intricacy of lung drug delivery, drug exposure, and effect, to ultimately optimize efficacy in antimicrobial therapy.

Advanced in vitro techniques, such as microfluidic chips or including more host cell types or host matrices, hold the potential to bridge the gap between simple in vitro experiments and clinical complexity. Nonetheless, infection as another component of in vitro systems can 
573 now follow the path of exploiting data towards the building of pharmacometrics models. This 574 field has used flow and dynamic infection systems for decades and, with increased 575 development, towards the use of hollow fiber model and multi-compartment models. This 576 review is a call for combining technologies, knowledge, computational power, and clinical 577 advances to build predictive in vitro models that integrate human host biology, the microbiome, 578 either associated or not to pathology, and the need for targeting drug delivery as a dynamic 579 interaction of these three major elements. 
580 Acknowledgments

581 CVMP was supported by the European Union's HORIZON 2020 Program under grant 582 agreement no. 642028 H2020-MSCA-ITN-2014, NABBA (Design, and Development of 583 advanced NAnomedicines to overcome Biological BArriers and to treat severe diseases). CK 584 was supported by BMBF (Bundesministerium für Bildung und Forschung/German Federal 585 Ministry of Education and Research, grant number: 031L0098) in the context of the Joint 586 Programming Initiative on Antimicrobial Resistance project "COMBINATORIALS." We 587 thank Dr. Ana Costa for the suggestions and comments, Olga Hartwig for the scientific 588 illustrations (graphic abstract and figure 1), and Dr. Alysia Cox for the English proofreading. 


\section{References}

[1] Institute of Health Metrix and Evaluations [IHME]. Findings from the Global Burden of Disease Study 2017. Seattle, WA: 2017.

[2] Peterson JW. Bacterial Pathogenesis - Medical Microbiology. Med Microbiol 1996. http://www.ncbi.nlm.nih.gov/books/NBK8526/.

[3] O’Neill J. Review on Antimicrobial Resistance Antimicrobial Resistance: Tackling a crisis for the health and wealth of nations. 2015.

[4] Perniss A, Liu S, Boonen B, Keshavarz M, Ruppert AL, Timm T, et al. Chemosensory Cell-Derived Acetylcholine Drives Tracheal Mucociliary Clearance in Response to Virulence-Associated Formyl Peptides. Immunity 2020;52:683-699.e11. https://doi.org/10.1016/j.immuni.2020.03.005.

[5] Chmiel JF, Davis PB. State of the art: why do the lungs of patients with cystic fibrosis become infected and why can't they clear the infection? Respir Res 2003;4:8. https://doi.org/10.1186/1465-9921-4-8.

[6] Moreau-Marquis S, Bomberger JM, Anderson GG, Swiatecka-Urban A, Ye S, O'Toole G a, et al. The DeltaF508-CFTR mutation results in increased biofilm formation by Pseudomonas aeruginosa by increasing iron availability. Am J Physiol Lung Cell Mol Physiol 2008;295:L25-37. https://doi.org/10.1152/ajplung.00391.2007.

[7] Mou H, Brazauskas K, Rajagopal J. Personalized medicine for cystic fibrosis: Establishing human model systems. Pediatr Pulmonol 2015;50:S14-23. https://doi.org/10.1002/ppul.23233.

[8] Wicha SG, Huisinga W, Kloft C. Translational pharmacometric evaluation of typical antibiotic broad-spectrum combination therapies against Staphylococcus aureus exploiting in vitro information. CPT Pharmacometrics Syst Pharmacol 2017;6:515-22. https://doi.org/10.1002/psp4.12197.

[9] Ding P, Wu H, Fang L, Wu M, Liu R. Transmigration and phagocytosis of macrophages in an airway infection model using four-dimensional techniques. Am J Respir Cell Mol Biol 2014;51:1-10. https://doi.org/10.1165/rcmb.2013-0390TE.

[10] Iqbal K, Broeker A, Nowak H, Rahmel T, Nussbaumer-Pröll A, Österreicher Z, et al. A pharmacometric approach to define target site-specific breakpoints for bacterial killing and resistance suppression integrating microdialysis, time-kill curves and heteroresistance data: A case study with moxifloxacin. Clin Microbiol Infect 2020. https://doi.org/10.1016/j.cmi.2020.02.013.

[11] Gloede J, Scheerans C, Derendorf H, Kloft C. In vitro pharmacodynamic models to 
determine the effect of antibacterial drugs. J Antimicrob Chemother 2009;65:186-201. https://doi.org/10.1093/jac/dkp434.

[12] Liu Q, Guan J, Qin L, Zhang X, Mao S. Physicochemical properties affecting the fate of nanoparticles in pulmonary drug delivery. Drug Discov Today 2020;25:150-9. https://doi.org/10.1016/j.drudis.2019.09.023.

[13] Knight DA, Rossi FM, Hackett TL. Mesenchymal stem cells for repair of the airway epithelium in asthma. Expert Rev Respir Med 2010;4:747-58. https://doi.org/10.1586/ers.10.72.

[14] Han S, Mallampalli RK. The Role of Surfactant in Lung Disease and Host Defense against. Ann Am Thorac Soc 2015;5:765-74. https://doi.org/10.1513/AnnalsATS.201411-507FR.

[15] Nahar K, Gupta N, Gauvin R, Absar S, Patel B, Gupta V, et al. In vitro, in vivo and ex vivo models for studying particle deposition and drug absorption of inhaled pharmaceuticals. Eur J Pharm Sci 2013;49:805-18. https://doi.org/10.1016/j.ejps.2013.06.004.

[16] Miller AJ, Spence JR. In Vitro Models to Study Human Lung Development, Disease and Homeostasis. Physiology 2017;32:246-60. https://doi.org/10.1152/physiol.00041.2016.

[17] Darquenne C, Fleming JS, Katz I, Martin AR. Bridging the Gap Between Science and Clinical Efficacy : J Aerosol Med Pulm Drug Deliv 2016;29:107-26. https://doi.org/10.1089/jamp.2015.1270.

[18] Acosta M, Muralidharan P, Meenach S, Hayes D, Children C. In Vitro Pulmonary Cell Culture in Pharmaceutical Inhalation Aerosol Delivery: 2-D, 3-D, and In Situ Bioimpactor Models. Curr Pharm Des 2016;22:2522-31. https://doi.org/10.2174/1381612822666160202142104.

[19] Lacroix G, Koch W, Larsen ST, Loret T, Zanetti F, Constant S, et al. Air - Liquid Interface In Vitro Models for Respiratory Toxicology Research : Appl Vitr Toxicol 2018;4:1-16. https://doi.org/10.1089/aivt.2017.0034.

[20] Hittinger M, Mell NA, Huwer H, Loretz B. Autologous Co-culture of Primary Human Alveolar Macrophages and Epithelial Cells for Investigating Aerosol Medicines . Part II : Evaluation of IL-10-loaded Microparticles for the Treatment of Lung Inflammation. Altern to Lab Anim ATLA 2016;44:349-60. https://doi.org/10.1177/026119291604400405.

[21] Fernandes C a, Vanbever R. Preclinical models for pulmonary drug delivery. Expert 
Opin Drug Deliv 2009;6:1231-45. https://doi.org/10.1517/17425240903241788.

[22] Kletting S, Barthold S, Repnik U, Griffiths G, Loretz B, Schneider-Daum N, et al. Coculture of human alveolar epithelial (hAELVi) and macrophage (THP-1) cell lines. ALTEX 2018;35:211-22. https://doi.org/10.14573/altex.1607191.

[23] de Souza Carvalho C, Daum N, Lehr CM. Carrier interactions with the biological barriers of the lung: Advanced in vitro models and challenges for pulmonary drug delivery. Adv Drug Deliv Rev 2014;75:129-40. https://doi.org/10.1016/j.addr.2014.05.014.

[24] Rothen-Rutishauser B, Mueller L, Blank F, Brandenberger C, Muehlfeld C, Gehr P. A newly developed in vitro model of the human epithelial airway barrier to study the toxic potential of nanoparticles. ALTEX Altern Zu Tierexperimenten 2008;25:191-6. https://doi.org/10.1517/17425255.4.8.1075.

[25] Kasper JY, Hermanns MI, Unger RE, Kirkpatrick CJ. A responsive human tripleculture model of the air-blood barrier: incorporation of different macrophage phenotypes. J Tissue Eng Regen Med 2017;11:1285-97. https://doi.org/10.1002/term.2032.

[26] Takai D, Nagase T, Shimizu T. New therapeutic key for cystic fibrosis: a role for lipoxins. Nat Immunol 2004;5:357-8. https://doi.org/10.1038/ni0404-357.

[27] de Jong PM, van Sterkenburg MAJA, Kempenaar JA, Dijkman JH, Ponec M. Serial culturing of human bronchial epithelial cells derived from biopsies. Vitr Cell Dev Biol - Anim J Soc Vitr Biol 1993;29:379-87. https://doi.org/10.1007/BF02633985.

[28] Awatade NT, Wong SL, Hewson CK, Fawcett LK, Kicic A, Jaffe A, et al. Human Primary Epithelial Cell Models: Promising Tools in the Era of Cystic Fibrosis Personalized Medicine. Front Pharmacol 2018;9:1-11. https://doi.org/10.3389/fphar.2018.01429.

[29] Pollard BS, Pollard HB. Induced pluripotent stem cells for treating cystic fibrosis: State of the science. Pediatr Pulmonol 2018;53:S12-29. https://doi.org/10.1002/ppul.24118.

[30] Kuehn A, Kletting S, De Souza Carvalho-Wodarz C, Repnik U, Griffiths G, Fischer U, et al. Human alveolar epithelial cells expressing tight junctions to model the air-blood barrier. ALTEX 2016;33:251-60. https://doi.org/10.14573/altex.1511131.

[31] Hittinger M, Juntke J, Kletting S, Schneider-Daum N, de Souza Carvalho C, Lehr CM. Preclinical safety and efficacy models for pulmonary drug delivery of antimicrobials with focus on in vitro models. Adv Drug Deliv Rev 2014:1-13. 
https://doi.org/10.1016/j.addr.2014.10.011.

[32] Nahar K, Gupta N, Gauvin R, Absar S, Patel B, Gupta V, et al. In vitro, in vivo and ex vivo models for studying particle deposition and drug absorption of inhaled pharmaceuticals. Eur J Pharm Sci 2013;49:805-18. https://doi.org/10.1016/j.ejps.2013.06.004.

[33] Huh D, Matthews BD, Mammoto A, Montoya-Zavala M, Hsin HY, Ingber DE. Reconstituting Organ-Level Lung Functions on a Chip. Science (80- ) 2010:1662-8. https://doi.org/10.1126/science.1188302.

[34] Bovard D, Sandoz A, Luettich K, Frentzel S, Iskandar A, Marescotti D, et al. A lung/liver-on-a-chip platform for acute and chronic toxicity studies. Lab Chip 2018;18:3814-29. https://doi.org/10.1039/c81c01029c.

[35] Ong HX, Traini D, Bebawy M, Young PM. Ciprofloxacin is actively transported across bronchial lung epithelial cells using a calu-3 air interface cell model. Antimicrob Agents Chemother 2013;57:2535-40. https://doi.org/10.1128/AAC.0030613.

[36] Marx U, Akabane T, Andersson TB, Baker E, Beilmann M, Beken S. Biology-Inspired Microphysiological Systems to Advance Patient Benefit and Animal Welfare in Drug Development. ALTEX Altern Zu Tierexperimenten 2020;37:365-94. https://doi.org/10.14573/altex.2001241.

[37] Elias-kirma S, Artzy-schnirman A, Das P, Heller-algazi M, Korin N, Sznitman J. In situ- Like Aerosol Inhalation Exposure for Cytotoxicity Assessment Using Airway-onChips Platforms. Front Bioeng Biotechnol 2020;8:1-13. https://doi.org/10.3389/fbioe.2020.00091.

[38] Sachs N, Papaspyropoulos A, Zomer-van Ommen DD, Heo I, Böttinger L, Klay D, et al. Long-term expanding human airway organoids for disease modeling. EMBO J 2019;38:1-20. https://doi.org/10.15252/embj.2018100300.

[39] Sakagami M. In vivo, in vitro and ex vivo models to assess pulmonary absorption and disposition of inhaled therapeutics for systemic delivery. Adv Drug Deliv Rev 2006;58:1030-60. https://doi.org/10.1016/j.addr.2006.07.012.

[40] Pezzulo AA, Starner TD, Scheetz TE, Traver GL, Tilley AE, Harvey B-G, et al. The air-liquid interface and use of primary cell cultures are important to recapitulate the transcriptional profile of in vivo airway epithelia. Am J Physiol Cell Mol Physiol 2010;300:L25-31. https://doi.org/10.1152/ajplung.00256.2010.

[41] Montefusco-Pereira CV, Horstmann JC, Ebensen T, Beisswenger C, Bals R, Guzmán 
CA, et al. P. aeruginosa infected 3D coculture of bronchial epithelial cells and macrophages at air-liquid interface for preclinical evaluation of anti-infectives. J Vis Exp 2020;in press.

[42] Luyts K, Napierska D, Dinsdale D, Klein SG, Serchi T, Hoet PHM. A coculture model of the lung-blood barrier: The role of activated phagocytic cells. Toxicol Vitr 2015;29:234-41. https://doi.org/10.1016/j.tiv.2014.10.024.

[43] Noguchi C, Umino T, Miyazaki Y, Jinta T, Usui Y, Yoshizawa Y. TGF- $\beta$ and glutathione promote tissue repair in cigarette smoke induced injury. J Med Dent Sci 2007;54:109-16. https://doi.org/10.11480/jmds.540115.

[44] Yonker LM, Mou H, Chu KK, Pazos MA, Leung H, Cui D, et al. Development of a Primary Human Co-Culture Model of Inflamed Airway Mucosa. Sci Rep 2017;7:1-12. https://doi.org/10.1038/s41598-017-08567-w.

[45] Blank F, Rothen-Rutishauser BM, Schurch S, Gehr P. An Optimized In Vitro Model of the Respiratory Tract Wall to Study Particle Cell Interactions . J Aerosol Med 2006;19:392-405. https://doi.org/10.1089/jam.2006.19.392.

[46] Costa A, de Souza Carvalho-Wodarz C, Seabra V, Sarmento B, Lehr CM. Triple coculture of human alveolar epithelium, endothelium and macrophages for studying the interaction of nanocarriers with the air-blood barrier. Acta Biomater 2019;91:23547. https://doi.org/10.1016/j.actbio.2019.04.037.

[47] Hermanns MI, Unger RE, Kehe K, Peters K, Kirkpatrick CJ. Lung epithelial cell lines in coculture with human pulmonary microvascular endothelial cells: development of an alveolo-capillary barrier in vitro. Lab Invest 2004;84:736-52. https://doi.org/10.1038/labinvest.3700081.

[48] Chowdhury F, Howat WJ, Phillips GJ, Lackie PM. Interactions between endothelial cells and epithelial cells in a combined cell model of airway mucosa: Effects on tight junction permeability. Exp Lung Res 2010;36:1-11. https://doi.org/10.3109/01902140903026582.

[49] Papritz M, Pohl C, Wübbeke C, Moisch M, Hofmann H, Hermanns MI, et al. Sidespecific effects by cadmium exposure: Apical and basolateral treatment in a coculture model of the blood-air barrier. Toxicol Appl Pharmacol 2010;245:361-9. https://doi.org/10.1016/j.taap.2010.04.002.

[50] Awatade NT, Uliyakina I, Farinha CM, Clarke LA, Mendes K, Solé A, et al. Measurements of Functional Responses in Human Primary Lung Cells as a Basis for Personalized Therapy for Cystic Fibrosis. EBioMedicine 2015;2:147-53. 
https://doi.org/10.1016/j.ebiom.2014.12.005.

[51] Araya J, Cambier S, Markovics J a, Wolters P, Jablons D, Hill A, et al. Squamous Metaplasia Amplifies Pathologic Epithelial-Mesenchymal Interactions in COPD Patients. J Clin Invest 2007;117:3551-62. https://doi.org/10.1172/JCI32526.

[52] Delhaes L, Monchy S, Fréalle E, Hubans C, Salleron J, Leroy S, et al. The airway microbiota in cystic fibrosis: A complex fungal and bacterial community-implications for therapeutic management. PLoS One 2012;7. https://doi.org/10.1371/journal.pone.0036313.

[53] Haswell LE, Hewitt K, Thorne D, Richter A, Gaça MD. Toxicology in Vitro Cigarette smoke total particulate matter increases mucous secreting cell numbers in vitro : A potential model of goblet cell hyperplasia. Toxicol Vitr 2010;24:981-7. https://doi.org/10.1016/j.tiv.2009.12.019.

[54] Fliegauf M, Sonnen AF, Kremer B, Henneke P. Mucociliary Clearance Defects in a Murine In Vitro Model of Pneumococcal Airway Infection 2013;8. https://doi.org/10.1371/journal.pone.0059925.

[55] Shambat SM, Chen P, Thu A, Hoang N, Bergsten H, Vandenesch F, et al. Modelling staphylococcal pneumonia in a human 3D lung tissue model system delineates toxinmediated pathology. Dis Model Mech 2015;8:1413-25. https://doi.org/10.1242/dmm.021923.

[56] Hittinger M, Juntke J, Kletting S, Schneider-Daum N, de Souza Carvalho C, Lehr CM. Preclinical safety and efficacy models for pulmonary drug delivery of antimicrobials with focus on in vitro models. Adv Drug Deliv Rev 2015;85:44-56. https://doi.org/10.1016/j.addr.2014.10.011.

[57] Elkington P, Lerm M, Kapoor N, Mahon R, Pienaar E, Huh D, et al. In Vitro Granuloma Models of Tuberculosis : Potential and Challenges. J Infect Dis 2019;219:1858-66. https://doi.org/10.1093/infdis/jiz020.

[58] Takayama K. In Vitro and Animal Models for SARS-CoV- 2 research Trends in Pharmacological Sciences. Trends Pharmacol Sci 2020;41:513-7. https://doi.org/10.1016/j.tips.2020.05.005.

[59] Cutting GR. Cystic fibrosis genetics: from molecular understanding to clinical application. Nat Rev Genet 2015;16:45-56. https://doi.org/10.1038/nrg3849.Cystic.

[60] Chen YW, Huang SX, De Carvalho ALRT, Ho SH, Islam MN, Volpi S, et al. A threedimensional model of human lung development and disease from pluripotent stem cells. Nat Cell Biol 2017;19:542-9. https://doi.org/10.1038/ncb3510. 
[61] Dechecchi MC, Nicolis E, Bezzerri V, Vella A, Colombatti M, Assael BM, et al. MPB-07 reduces the inflammatory response to Pseudomonas aeruginosa in cystic fibrosis bronchial cells. Am J Respir Cell Mol Biol 2007;36:615-24. https://doi.org/10.1165/rcmb.2006-02000C.

[62] Greene CM, Carroll TP, Smith SGJ, Taggart CC, Devaney J, Griffin S, et al. TLRInduced Inflammation in Cystic Fibrosis and Non-Cystic Fibrosis Airway Epithelial Cells. J Immunol 2014;174:1638-46. https://doi.org/10.4049/jimmunol.174.3.1638.

[63] Lehmann AD, Daum N, Bur M, Lehr CM, Gehr P, Rothen-Rutishauser BM. An in vitro triple cell coculture model with primary cells mimicking the human alveolar epithelial barrier. Eur J Pharm Biopharm 2011;77:398-406. https://doi.org/10.1016/j.ejpb.2010.10.014.

[64] Moreau-Marquis S, Redelman C V, Stanton B a, Anderson GG. Coculture models of Pseudomonas aeruginosa biofilms grown on live human airway cells. J Vis Exp 2010:2-5. https://doi.org/10.3791/2186.

[65] LaFayette SL, Houle D, Beaudoin T, Wojewodka G, Radzioch D, Hoffman LR, et al. Cystic fibrosis-adapted Pseudomonas aeruginosa quorum sensing lasR mutants cause hyperinflammatory responses . Sci Adv 2015;1:e1500199. https://doi.org/10.1126/sciadv.1500199.

[66] Meek MEB, Lipscomb JC. Gaining acceptance for the use of in vitro toxicity assays and QIVIVE in regulatory risk assessment. Toxicology 2015;332:112-23. https://doi.org/10.1016/j.tox.2015.01.010.

[67] Graef F, Richter R, Fetz V, Murgia X, De Rossi C, Schneider-Daum N, et al. In Vitro Model of the Gram-Negative Bacterial Cell Envelope for Investigation of AntiInfective Permeation Kinetics. ACS Infect Dis 2018;4:1188-96. https://doi.org/10.1021/acsinfecdis.7b00165.

[68] Vandeplassche E, Sass A, Lemarcq A, Dandekar AA, Coenye T, Crabbé A. In vitro evolution of Pseudomonas aeruginosa AA2 biofilms in the presence of cystic fibrosis lung microbiome members. Sci Rep 2019;9:1-14. https://doi.org/10.1038/s41598-01949371-y.

[69] Müller L, Murgia X, Siebenbürger L, Börger C, Schwarzkopf K, Sewald K, et al. Human airway mucus alters susceptibility of Pseudomonas aeruginosa biofilms to tobramycin, but not colistin. J Antimicrob Chemother 2018;73:2762-9. https://doi.org/10.1093/jac/dky241.

[70] Ceri H, Olson ME, Stremick C, Read RR, Morck D, Buret A. The Calgary Biofilm 
Device: New technology for rapid determination of antibiotic susceptibilities of bacterial biofilms. J Clin Microbiol 1999;37:1771-6. https://doi.org/10.1128/jcm.37.6.1771-1776.1999.

[71] Bergen PJ, Tsuji BT, Bulitta JB, Forrest A, Jacob J, Sidjabat HE, et al. Synergistic Killing of Multidrug-Resistant Pseudomonas aeruginosa at Multiple Inocula by Colistin Combined with Doripenem in an In Vitro Pharmacokinetic / Pharmacodynamic Model $\square$. Antimicrob Agents Chemother 2011;55:5685-95. https://doi.org/10.1128/AAC.05298-11.

[72] Pichereau S, Pantrangi M, Couet W, Badiou C, Lina G, Shukla SK, et al. Simulated Antibiotic Exposures in an In Vitro Hollow-Fiber Infection Model Influence Toxin Gene Expression and Production in Community-Associated Methicillin-Resistant Staphylococcus aureus. Antimicrob Agents Chemother 2012;56:140-7. https://doi.org/10.1128/AAC.05113-11.

[73] Stanton BA, Coutermarsh B, Barnaby R, Hogan D. Pseudomonas aeruginosa reduces VX-809 stimulated F508del-CFTR chloride secretion by airway epithelial cells. PLoS One 2015;10:1-13. https://doi.org/10.1371/journal.pone.0127742.

[74] Barkal LJ, Procknow CL, Álvarez-garcía YR, Niu M, Jiménez-torres JA, Brockmanschneider RA, et al. Microbial volatile communication in human organotypic lung models. Nat Commun 2017;1770. https://doi.org/10.1038/s41467-017-01985-4.

[75] Gloede J, Scheerans C, Derendorf H, Kloft C. In vitro pharmacodynamic models to determine the effect of antibacterial drugs. J Antimicrob Chemother 2009;65:186-201. https://doi.org/10.1093/jac/dkp434.

[76] Scheerans C, Wicha SG, Michael J, Derendorf H, Kloft C. Concentration-response studies and modelling of the pharmacodynamics of linezolid: Staphylococcus aureus versus Enterococcus faecium. Int J Antimicrob Agents 2015;45:54-60. https://doi.org/10.1016/j.ijantimicag.2014.07.028.

[77] Odenholt I, Cars O. Pharmacodynamics of moxifloxacin and levofloxacin against Streptococcus pneumoniae, Staphylococcus aureus, Klebsiella pneumoniae and Escherichia coli: Simulation of human plasma concentrations after intravenous dosage in an in vitro kinetic model. J Antimicrob Chemother 2006;58:960-5. https://doi.org/10.1093/jac/dk1356.

[78] Khan DD, Lagerb??ck P, Cao S, Lustig U, Nielsen EI, Cars O, et al. A mechanismbased pharmacokinetic/pharmacodynamic model allows prediction of antibiotic killing from MIC values for WT and mutants. J Antimicrob Chemother 2015;70:3051-60. 
https://doi.org/10.1093/jac/dkv233.

[79] Jacobs M, Grégoire N, Couet W, Bulitta JB. Distinguishing Antimicrobial Models with Different Resistance Mechanisms via Population Pharmacodynamic Modeling. PLoS Comput Biol 2016;12:e1004782. https://doi.org/10.1371/journal.pcbi.1004782.

[80] Thorsted A, Tano E, Kaivonen K, Sjölin J, Friberg LE, Nielsen EI. Extension of pharmacokinetic/pharmacodynamic (PK/PD) time-kill studies to include LPS/endotoxin release from E. coli exposed to cefuroxime . Antimicrob Agents Chemother 2020;64:1-12. https://doi.org/10.1128/aac.02070-19.

[81] Rostami-Hodjegan A. Physiologically based pharmacokinetics joined with in vitro-in vivo extrapolation of ADME: A marriage under the arch of systems pharmacology. Clin Pharmacol Ther 2012;92:50-61. https://doi.org/10.1038/clpt.2012.65.

[82] Thieme L, Hartung A, Tramm K, Klinger-Strobel M, Jandt KD, Makarewicz O, et al. MBEC Versus MBIC: The Lack of Differentiation between Biofilm Reducing and Inhibitory Effects as a Current Problem in Biofilm Methodology. Biol Proced Online 2019;21:1-5. https://doi.org/10.1186/s12575-019-0106-0.

[83] Zhao M, Lepak AJ, Andes DR. Animal models in the pharmacokinetic / pharmacodynamic evaluation of antimicrobial agents. Bioorg Med Chem 2016;24:6390-400.

[84] Mouton JW, Dudley MN, Cars O, Derendorf H, Drusano GL. Standardization of pharmacokinetic/pharmacodynamic (PK/PD) terminology for anti-infective drugs: An update. J Antimicrob Chemother 2005;55:601-7. https://doi.org/10.1093/jac/dki079.

[85] Lin Y-W, Zhou QT, Cheah S-E, Zhao J, Chen K, Wang J, et al. Pharmacokinetics / Pharmacodynamics of Pulmonary Delivery of Colistin against Pseudomonas aeruginosa in a Mouse. Antimicrob Agents Chemother 2017;61:1-11.

[86] Okusanya OO, Bhavnani SM, Hammel JP, Forrest A, Bulik CC, Ambrose PG, et al. Evaluation of the pharmacokinetics and pharmacodynamics of liposomal amikacin for inhalation in cystic fibrosis patients with chronic pseudomonal infections using data from two phase 2 clinical studies. Antimicrob Agents Chemother 2014;58:5005-15. https://doi.org/10.1128/AAC.02421-13.

[87] Committee for Human Medicinal Products (CHMP). Guideline on the use of pharmacokinetics and pharmacodynamics in the development of antibacterial medicinal products. Eur Med Agency 2016;44:1-21. https://doi.org/10.1080/09500690010006473.

[88] Jusko WJ. Moving from basic toward systems pharmacodynamic models. J Pharm Sci 
2013;102:2930-40. https://doi.org/10.1002/jps.23590.

[89] Rathi C, Lee RE, Meibohm B. Translational PK/PD of Anti-Infective Therapeutics. Drug Discov Today Technol 2016;21-22:41-9. https://doi.org/10.1016/j.ddtec.2016.08.004.

[90] Borghardt JM, Kloft C, Sharma A. Inhaled Therapy in Respiratory Disease: The Complex Interplay of Pulmonary Kinetic Processes. Can Respir J 2018;2018. https://doi.org/10.1155/2018/2732017.

[91] Borghardt JM, Weber B, Staab A, Kloft C. Pharmacometric Models for Characterizing the Pharmacokinetics of Orally Inhaled Drugs. AAPS J 2015;17:853-70. https://doi.org/10.1208/s12248-015-9760-6.

[92] Min KA, Talattof A, Tsume Y, Stringer KA, Yu JY, Lim DH, et al. The extracellular microenvironment explains variations in passive drug transport across different airway epithelial cell types. Pharm Res 2013;30:2118-32. https://doi.org/10.1007/s11095-0131069-5.

[93] European Society of Clinical Microbiology and Infectious Diseases. EUCAST: Clinical breakpoints and dosing of antibiotics. EUCAST, Eur Comm Antimicrob Susceptibility Test 2019:1.

[94] Trivedi A, Lee RE, Meibohm B. Applications of pharmacometrics in the clinical development and pharmacotherapy of anti-infectives. Expert Rev Clin Pharmacol 2013;6:159-70. https://doi.org/10.1586/ecp.13.6.

[95] Diaz Perez A, Kougl K, Vasicek TW, Liyanage R, Lay J, Stenken JA. Microdialysis sampling of quorum sensing homoserine lactones during biofilm formation. Anal Chem 2019;91:3964-70. https://doi.org/10.1021/acs.analchem.8b05168.

[96] Sou T, Kukavica-Ibrulj I, Levesque R, Friberg L, Bergström C. Model-informed drug development in pulmonary delivery: Preclinical pharmacokinetic-pharmacodynamic modelling for evaluation of treatments against chronic Pseudomonas aeruginosa lung infections. Mol Pharm 2020:Jan 17. https://doi.org/10.1021/acs.molpharmaceut.9b00968.

[97] Torres BGS, Helfer VE, Bernardes PM, Macedo AJ, Nielsen EI, Friberg LE, et al. Population pharmacokinetic modeling as a tool to characterize the decrease in biofilm lung infection in Wistar rats. Antimicrob Agents Chemother 2017;61:1-14.

[98] Haagensen J, Verotta D, Huang L, Engel J, Spormann AM, Yang K. Spatiotemporal pharmacodynamics of meropenem- and tobramycin-treated Pseudomonas aeruginosa biofilms. J Antimicrob Chemother 2017;72:3357-65. 
https://doi.org/10.1093/jac/dkx288.

930 [99] Rees VE, Yadav R, Rogers KE, Bulitta JB, Wirth V, Oliver A, et al. Meropenem combined with ciprofloxacin combats hypermutable pseudomonas aeruginosa from respiratory infections of cystic fibrosis patients. Antimicrob Agents Chemother 2018;62:1-12. https://doi.org/10.1128/AAC.01150-18.

934 [100] Silveira SA, Shorr AF. Critical Parameters for the Development of Novel Therapies for Severe and Resistant Infections - A Case Study on CAL02, a Non-Traditional. Antibiotics 2020;9.

937 [101] Jesaitis AJ, Franklin MJ, Berglund D, Sasaki M, Lord CI, Bleazard JB, et al. Compromised Host Defense on Pseudomonas aeruginosa Biofilms: Characterization of Neutrophil and Biofilm Interactions . J Immunol 2003;171:4329-39. https://doi.org/10.4049/jimmunol.171.8.4329.

941 
Figure 1. Evolution of in vitro models for the investigation of lung host-microbe interactions. 1A) Cell procurement and its modes of cultivation. 1B) Transwell systems with the integration of immune cells. 1C) Models to mimic air-to-blood transport through cell layers and adaptation to its natural structures. 1D) Infection models with bacterial biofilm growth using $P$. aeruginosa. 1E) Use of scaffolds to develop longer and mature bacterial biofilms. 1F) Evolution of infection models combining other microbes relevant to chronic infection as $S$. aureus, the complex B. cenocepacia, and the respiratory syncytial virus.

Figure 2. The factors and information to build an essential PK/PD model for new antiinfectives.

Table 1. Cellular in vitro systems that overlap drug efficacy studies (including aerosol delivery), microbiological, and human cell biology parameters towards the possibility of new PK/PD models.

Abbreviations: Air-liquid interface (ALI), Airways surface liquid (ASL), Biological safety laboratory (BSL), Ciprofloxacin (CIP), Confocal laser scanning microscopy (CLSM), Cystic fibrosis (CF), Extracellular matrix (ECM), Ethylenediaminetetraacetic acid (EDTA), Hollow fiber model (HFM), Human pluripotent stem cells (hPSCs), Isolated perfused lung (IPL), Liquid covered conditions (LCC), Methicillin-Resistant Staphylococcus aureus strain (MRSA), Minimal biofilm eradication concentration (MBEC), Multidrug resistance-associated proteins (MRPs), Multiplicity of infection (MOI), Organic cation transporters (OCTs), Organic anion-transporting polypeptides (OATPs), Polydimethylsiloxane (PDMS), Polyethyleneglycol (PEG), Plasmid deoxyribonucleic acid (pDNA), Pharmacodynamics (PD), Pharmacokinetics (PK), Pseudomonas aeruginosa (PA), Respiratory syncytial virus (RSV), Sodium fluorescein (NaFlu), Staphylococcus aureus (SA), Staphylococcus newman (SN), Streptococcus salavari (SS).

Table 2. In vitro bacteria-infected systems that overlap drug efficacy studies (including aerosol delivery), microbiological and human cell biology parameters towards the possibility of new PK/PD models. 
975 Abbreviations: Air-liquid interface (ALI), Airways surface liquid (ASL), Biological safety 976 laboratory (BSL), Ciprofloxacin (CIP), Confocal laser scanning microscopy (CLSM), Cystic 977 fibrosis (CF), Extracellular matrix (ECM), Ethylenediaminetetraacetic acid (EDTA), Hollow 978 fiber model (HFM), Human pluripotent stem cells (hPSCs), Isolated perfused lung (IPL), 979 Liquid covered conditions (LCC), Methicillin-Resistant Staphylococcus aureus strain 980 (MRSA), Minimal biofilm eradication concentration (MBEC), Multidrug resistance-associated 981 proteins (MRPs), Multiplicity of infection (MOI), Organic cation transporters (OCTs), Organic 982 anion-transporting polypeptides (OATPs), Polydimethylsiloxane (PDMS), Polyethyleneglycol 983 (PEG), Plasmid deoxyribonucleic acid (pDNA), Pharmacodynamics (PD), Pharmacokinetics 984 (PK), Pseudomonas aeruginosa (PA), Respiratory syncytial virus (RSV), Sodium fluorescein 985 (NaFlu), Staphylococcus aureus (SA), Staphylococcus newman (SN), Streptococcus salavari 986 (SS).

987

988 Table 3. Recent reports on the combination of in vivo and in vitro models to inform 989 pharmacometrics mathematical models. Pharmacometrics approach: Noncompartmental 990 analysis (NCA), pharmacokinetic/pharmacodynamic (PK/PD), population pharmacokinetic 991 (popPK), physiologically-based pharmacokinetic (PB)PK.

992 Further abbreviations: Acylhomoserine lactones (AHLs), Acyl-oxohomoserine lactones 993 (AOHLs), ciprofloxacin (CIP), colony-forming unit (CFU), cystic fibrosis (CF), epithelial 994 lining fluid (ELF), Escherichia coli (E. coli), grams (g), green fluorescent protein (GFP), 995 Hollow-fiber infection model (HFIM), High-performance Liquid Chromatography with 996 tandem mass spectrometry (HPLC-MS/MS), hours (h), intravenous (i.v.), mechanism-based 997 modeling (MBM), meropenem (MEM), milligrams (mg), milligrams/kilograms (mg/kg), 998 milligrams/liters (mg/L), minimum inhibitory concentration (MIC), nanograms/milliliter 999 (ng/mL), parts per billion (ppb), pharmacokinetics (PK), Staphylococcus aureus (S. aureus), 1000 Vibrio harveyi (V. harveyi). 